\title{
A DEMONSTRATION OF A POSSIBLE MECHANISM OF SYMPATRIC DIVERGENCE USING SIMULATION TECHNIQUES
}

\author{
S. H. JAMES \\ Botany Department, University of Western Australia
}

Received 26.vii. 69

\section{INTRODUCTION}

Chromosome repatterning, or the fixation of chromosome structural differences between infraspecific taxa, is an important but unsettled problem in evolutionary biology.

The purpose of this paper is to illustrate some situations of obvious biological validity in which natural selection will fix chromosomal rearrangements (e.g. interchanges) in populations sharing a potentially common gene pool. In effect, a model is described in which, following significant genetic differentiation, partial sympatry (migration) elicits the fixation of structural rearrangements in the separate infraspecific entities so that the significant differences between the entities will be conserved.

\section{DesGription OF THE MODEL}

Consider two closely adjacent populations, Population 1 and Population 2, which are separately and uniquely adapted to their individual environments. It is immaterial to the model as to whether this primary differentiation has been established during a previous period of allopatry, through disruptive selection under sympatric conditions, or through the genetic mobility of population isolates engendered by dispersive stochastic processes. Necessary conditions for this model are that between population migration occurs and that interpopulational hybrids show substantial segregational sterility.

Population 1 is made up of individuals who share a common gene pool which is uniquely integrated providing a maximum adaptation to the environment. Each individual in Population 1 is composed of two genomes drawn at random from the gametic pool generated by the breeding adults of the population. Let $A_{1}$ be the standard genome uniquely characteristic of Population 1. The individuals of Population 1 are thus $A_{1} A_{1}$ homogenomes, and must exhibit relationally balanced genetic architecture (Mather, 1943). Similarly, the individuals of Population 2 are $A_{2} A_{2}$ homogenomes, and also exhibit a relationally balanced genetic architecture. Let there be no overt chromosome structural rearrangements differentiating the two populations so that the interpopulational hybrid, $A_{1} A_{2}$, although heterogenomic, is homokaryotypic. Regardless of the vigour of this $A_{1} A_{2}$ interpopulational hybrid, let it be less fit than the standard homogenomes because of segregational sterility. That is, although there may be a good heterotic interaction between the two genomes, relational balance along with genomic integrity is destroyed at gametogenesis.

In Population 1, say, let a chromosome mutation (interchange) occur such that a variant $A_{3}$ is produced. 
$A_{1}$ is equivalent to $A_{3}$ genetically, but not structurally. $A_{1} A_{3}$ homogenomic heterokaryotypes will be partly infertile because of meiotic nondisjunction, and therefore less fit than the homokaryotypes $A_{1} A_{1}$ and $A_{3} A_{3}$.

Similarly, in Population 2, let a chromosome mutation (interchange) occur such that the variant genome $A_{4}$ is produced. The $A_{2} A_{4}$ homogenomic heterokaryotypes are, again, at a selective disadvantage relative to the $A_{2} A_{2}$ and $A_{4} A_{4}$ homokaryotypes.

The interpopulational hybrids possible are all heterogenomic and subject to segregational sterility. These hybrids may be homokaryotypic $\left(A_{1} A_{2}\right)$, or heterokaryotypic of a greater $\left(A_{3} A_{4}\right)$ or lesser $\left(A_{1} A_{4}, A_{2} A_{3}\right)$ extent. The heterokaryotypy of these interpopulation hybrids is of potential adaptive utility since it may reduce the segregational sterility of the heterogenomes. The course of any evolutionary episode will be determined by the parameters defined in the following table (a panmictic breeding system is envisaged).

Variable

Coefficient of Fitness for $A_{1} A_{1}$

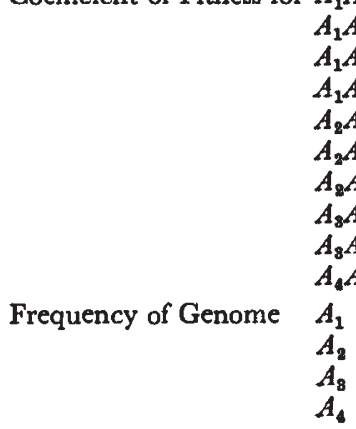

Migration Rate
In Population 1

$a$
$b$
$c$
$d$
$e$
$f$
$g$
$h$
$i$
$j$
$p_{1}$
$q_{1}$
$r_{1}$
$s_{1}$
$m_{1}$

In Population 2

$k$
$l$
$m$
$n$
$o$
$p$
$q$
$r$
$s$
$t$
$p_{2}$
$q_{2}$
$r_{2}$
$s_{2}$
$m_{2}$

The frequencies of the genomes $A_{1}, A_{2}, A_{3}$, and $A_{4}$, in each population in the next generation, viz. $p_{1}^{\prime}, q_{1}^{\prime}, r_{1}^{\prime}, s_{1}^{\prime}, p_{2}^{\prime}, q_{2}^{\prime}, r_{2}^{\prime}$ and $s_{2}^{\prime}$, are readily computed from the following equations:

$$
\begin{aligned}
& p_{1}^{\prime}=\frac{1}{\alpha}\left[m_{1} p_{2}\left(k p_{2}+l q_{2}+m r_{2}+n s_{2}\right)+\left(1-m_{1}\right) p_{1}\left(a p_{1}+b q_{1}+c r_{1}+d s_{1}\right)\right] \\
& q_{1}^{\prime}=\frac{1}{\alpha}\left[m_{1} q_{2}\left(l p_{2}+o q_{2}+p r_{2}+q s_{2}\right)+\left(1-m_{1}\right) q_{1}\left(b p_{1}+e q_{1}+f r_{1}+g s_{1}\right)\right] \\
& r_{1}^{\prime}=\frac{1}{\alpha}\left[m_{1} r_{2}\left(m p_{2}+p q_{2}+r r_{2}+s s_{2}\right)+\left(1-m_{1}\right) r_{1}\left(c p_{1}+f q_{1}+h r_{1}+i s_{1}\right)\right] \\
& s_{1}^{\prime}=\frac{1}{\alpha}\left[m_{1} s_{2}\left(n p_{2}+q q_{2}+s r_{2}+t s_{2}\right)+\left(1-m_{1}\right) s_{1}\left(d p_{1}+g q_{1}+i r_{1}+j s_{1}\right)\right] \\
& p_{2}^{\prime}=\frac{1}{\beta}\left[m_{2} p_{1}\left(a p_{1}+b q_{1}+c r_{1}+d s_{1}\right)+\left(1-m_{2}\right) p_{2}\left(k p_{2}+l q_{2}+m r_{2}+n s_{2}\right)\right] \\
& q_{2}^{\prime}=\frac{1}{\beta}\left[m_{2} q_{1}\left(b p_{1}+e q_{1}+f r_{1}+g s_{1}\right)+\left(1-m_{2}\right) q_{2}\left(l p_{2}+o q_{2}+p r_{2}+q s_{2}\right)\right] \\
& r_{2}^{\prime}=\frac{1}{\beta}\left[m_{2} r_{1}\left(c p_{1}+f q_{1}+h r_{1}+i s_{1}\right)+\left(1-m_{2}\right) r_{2}\left(m p_{2}+p q_{2}+r r_{2}+s s_{2}\right)\right] \\
& s_{2}^{\prime}=\frac{1}{\beta}\left[m_{2} s_{1}\left(d p_{1}+g q_{1}+i r_{1}+j s_{1}\right)+\left(1-m_{2}\right) s_{2}\left(n p_{2}+q q_{2}+s r_{2}+t s_{2}\right)\right]
\end{aligned}
$$




$$
\begin{aligned}
\text { where } \alpha= & m_{1}\left(k p_{2}^{2}+2 l p_{2} q_{2}+2 m p_{2} r_{2}+2 n p_{2} s_{2}+o q_{2}^{2}+2 p q_{2} r_{2}+2 q q_{2} s_{2}+r r_{2}^{2}+2 s r_{2} s_{2}\right. \\
& \left.+t s_{2}^{2}\right)+\left(1-m_{1}\right)\left(a p_{1}^{2}+2 b p_{1} q_{1}+2 c p_{1} r_{1}+2 d p_{1} s_{1}+e q_{1}^{2}+2 f q_{1} r_{1}+2 g q_{1} s_{1}\right. \\
& \left.+h r_{1}^{2}+2 i r_{1} s_{1}+j s_{1}^{2}\right) ; \\
\beta= & m_{2}\left(a p_{1}^{2}+2 b p_{1} q_{1}+2 c p_{1} r_{1}+2 d p_{1} s_{1}+e q_{1}^{2}+2 f q_{1} r_{1}+2 g q_{1} s_{1}+h r_{1}^{2}+2 i r_{1} s_{1}\right. \\
& \left.+j s_{1}^{2}\right)+\left(1-m_{2}\right)\left(k p_{2}^{2}+2 l p_{2} q_{2}+2 m p_{2} r_{2}+2 n p_{2} s_{2}+o q_{2}^{2}+2 p q_{2} r_{2}\right. \\
& \left.+2 q q_{2} s_{2}+r r_{2}^{2}+2 s r_{2} s_{2}+t s_{2}^{2}\right) ; \\
m_{1}= & \text { the migration rate affecting Population 1 (Population 1 zygotes } \\
& \text { are derived each generation by the random combination of } \\
& \text { gametes in a pool to which Population 2 contributes } m_{1} \text { and } \\
& \text { Population 1 contributes }\left(1-m_{1}\right) \text { randomly drawn gametes); }
\end{aligned}
$$

and $m_{2}=$ the migration rate affecting Population 2.

Thus, by assigning values to the 20 fitness coefficients, to the two migration rates and to the eight initial genome frequencies, it is possible to simulate over a sequence of successive generations, a variety of situations of cytoevolutionary interest.

\section{First series: With pREAdaptation}

\section{(a) Design of experiments}

Let us assume that the species is preadapted to exploit the chromosome mutation involved. On this basis, we may deduce a subjective but logical array of fitness coefficients for the case where interchange mutants are considered, thus :

(1) $A_{1} A_{1}$ and $A_{3} A_{3}$ in Population 1 are homogenomic homokaryotypes residing in the environment to which they are maximally adapted. Under these conditions their fitness should be unity.

Thus, say $a=1 \cdot 0, h=1.0$ and also, by similar reasoning, $0=1 \cdot 0, t=1 \cdot 0$.

(2) The homogenomic homokaryotypes if residing in environments to which they are not maximally adapted will have a fitness coefficient of something less than unity.

Thus, say $e=0.5, j=0.5, k=0.5$ and $r=0.5$.

(3) The homogenomic heterokaryotypes, $A_{1} A_{3}$ and $A_{2} A_{4}$, residing in the environment to which their genotypes maximally adapted them, will, however, be incompletely fertile because of the non-disjunction of their interchange catenations at meiosis. But, since the species has distal chiasma localisation it is preadapted for directed segregation and the fitness coefficient will be high.

Thus, say $c=0.9, q=0.9$.

(4) The homogenomic heterokaryotypes residing in environments to which they are not adapted will, because of non-disjunction, have a fitness below that of the corresponding homokaryotypes.

Thus, say $m=0.4, g=0.4$.

(5) The heterogenomic homokaryotypes, $A_{1} A_{2}$, are able to live in either environment, but they exhibit significant sterility because of segregational effects.

Thus, say $b=0 \cdot 5, l=0.5$.

(6) In heterogenomes, heterokaryotypy will be advantageous in decreasing the degree of segregational sterility. Where the heterogenome is 
heterozygous for a single interchange, as in $A_{1} A_{4}$ and $A_{2} A_{3}$, the conservative effect of the structural heterozygosity will not be as pronounced as in the double structural heterozygotes, $A_{3} A_{4}$.

Thus, say $d=0.7, n=0.7, f=0.7, p=0.7$ and $i=0.8$, $s=0.8$.

Migration rates can be chosen arbitrarily to simulate different biological situations. In these experiments, eight population pairs were initiated with migrational interactions of

$$
\begin{aligned}
& m_{1}=0.0 ; m_{2}=0.05,0.10,0.20,0.50 \\
& m_{1}=m_{2} ; m_{2}=0.05,0.10,0.20,0.50
\end{aligned}
$$$$
\left.\begin{array}{r}
\text { TOP ROW } \\
\text { BOTTOM ROW }
\end{array}\right\} \text { (fig. 2). }
$$

In all cases, the population pairs were initiated with genome frequencies arbitrarily chosen as

$$
\begin{aligned}
& p_{1}=0.9, q_{1}=0.0, r_{1}=0.1, s_{1}=0.0 \\
& p_{2}=0.0, q_{2}=0.9, r_{2}=0.0, s_{2}=0.1
\end{aligned}
$$

and the populations were run over one hundred consecutive generations.

The results of the simulations are presented in graphical form, and a key common to all graphs is presented in fig. 1. The results of the eight population pairs observed in this first series are presented in fig. 2.

Any individual population can be referred to by naming the figure number, the graph number and the population number. Thus, the top half of the bottom right graph in fig. 2 may be named population $(2,8,1)$.

The individual graphs, or population pairs, may also be so named (e.g. graph $(2,8))$.

\section{(b) Results of the experiments}

It is verified in population $(2,1,1)$ and in $(2,2,1),(2,3,1)$ and $(2,4,1)$, that where no migration into a population occurs and where there is no inherent selective advantage favouring one or other chromosome arrangement or the heterozygote apart from the heterozygote inferiority due to the sterility associated with non-disjunction, natural selection will lead to the elimination of the chromosome arrangement initially present in lowest frequency.

Population $(2,1,2)$ demonstrates that at low migrational intensities, a stable polymorphism may be achieved wherein migration pressure is balanced against selection pressure.

Population $(2,2,2)$ is a recipient population receiving a critical influx of immigrants. In this situation, the newly arisen chromosome mutation $A_{4}$ becomes an adaptive arrangement in that it resists the breakdown of genomic integrity in the interpopulational hybrids. Natural selection elicits the fixation of this more conservative genome. Clearly, this simulates a situation in which chromosome repatterning may occur.

Populations $(2,3,2)$ and $(2,4,2)$ are recipient populations swamped by the immigrants. That is, even though $A_{2}$ is a genome more highly adapted to the environment of Population 2 than is $A_{1}$, migration pressure from Population 1 leads to the replacement of $A_{2}$ by $A_{1}$. This simulates the situation confronting populations at the periphery of a species range. Environments to which it is not adapted limit the species distribution, and at the species 


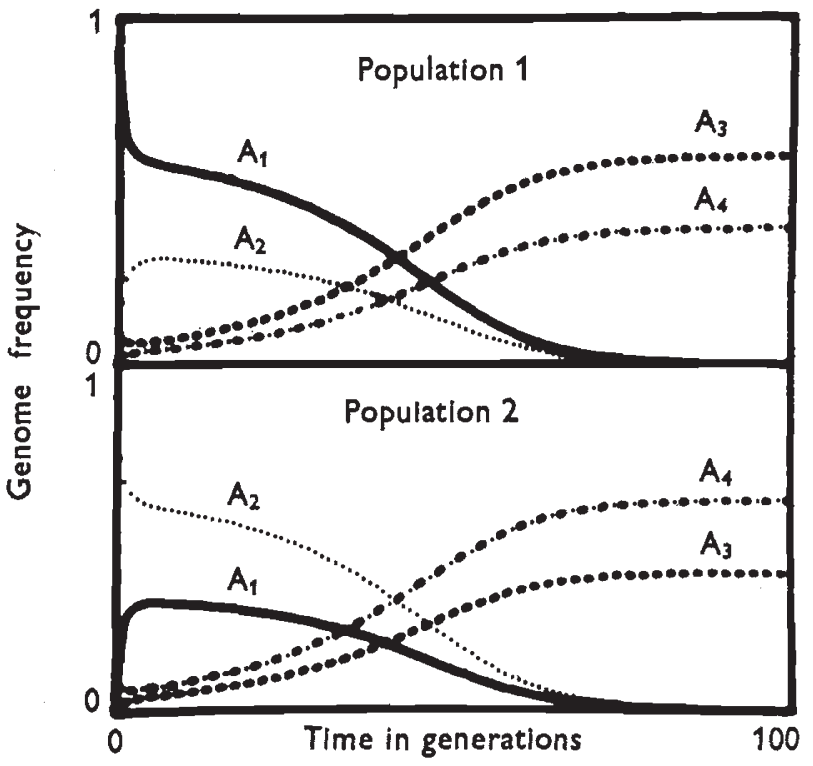

Fig. 1.-The course of an evolutionary episode as simulated by the model described. Two populations are involved; the upper half of the figure represents Population 1, the lower half Population 2. The vertical axis represents genome frequency ranging from 0.0 to 1.0 for each population; the horizontal axis represents time, ranging from Generation 0 to Generation 100. The lines represent the progressive changes in genome frequencies during the 100-generation episode, and a key to the genomes is provided in the figure. The parameters determining the outcome of the evolutionary episode are the fitness coefficients $(a, b, c, d, e, f, g, h, i, j, k, l, m, n, o, p, q, r, s, t)$, the migration rates $\left(m_{1}\right.$ and $\left.m_{2}\right)$ and the initial genome frequencies $\left(p_{1}, q_{1}, r_{1}, s_{1}, p_{2}, q_{2}, r_{2}\right.$, and $\left.s_{2}\right)$, the values of each being stated in the "Design of Experiments" for each Series.
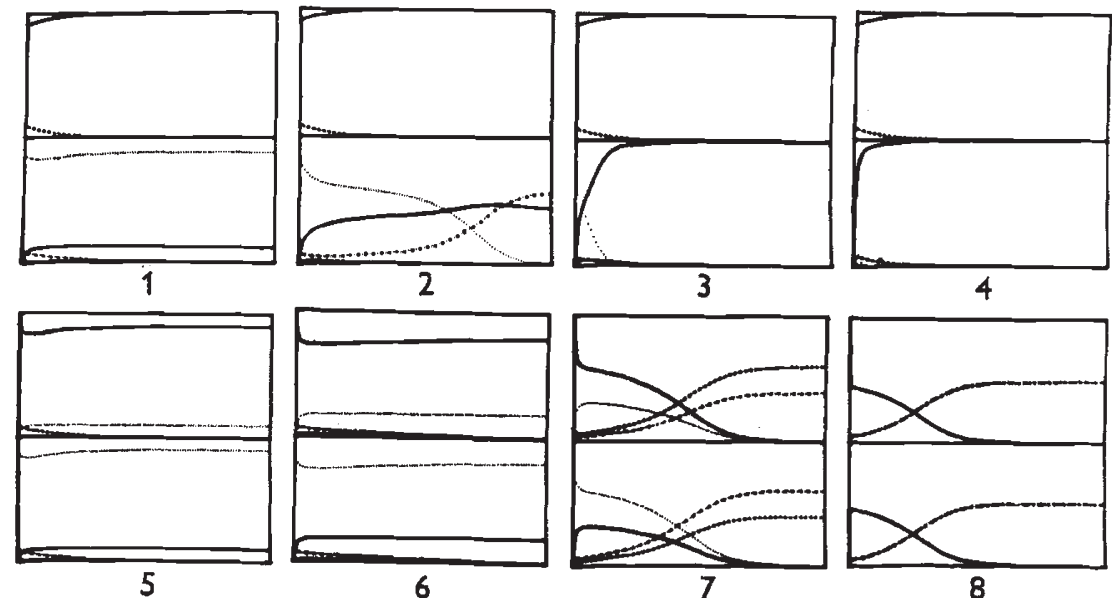

Frg. 2.-The eight episodes simulated in the First Series. See text for fitness coefficients deduced on the basis of preadaptation for interchange exploitation. In the top row, $m_{1}=0$; in the bottom row, $m_{1}=m_{2}$. By columns, moving left to right, $m_{2}=0.05$, $0 \cdot 1,0 \cdot 2,0.5$. The initial genome frequencies are $p_{1}=0.9, q_{1}=0.0, r_{1}=0.1, s_{1}=0.0$ and $p_{2}=0.0, q_{2}=0.9, r_{2}=0.0, s_{2}=0.1$. 
border genetic recombination engendered by sexual reproduction may establish new gene arrays conferring increased adaptation upon their carriers. However, the swamping effect of heavy migration from the body of the species into the peripheral isolate will lead to the elimination of any such newly adapted genome. (Note that the migration rate, however, is subject to endogenous control; changes in the breeding system may preadapt the population to resist otherwise irresistible migrational influxes.)

The migration rates between the populations in pairs $(2,5),(2,6),(2,7)$ and $(2,8)$ are equal. This avoids the swamping of one population by the other. Again, at a critical level of migrational interaction, natural selection elicits the fixation of the most conservative genomes in the separate populations. In the pair $(2,8)$, the frequencies of $A_{1}$ and $A_{2}$ and of $A_{3}$ and $A_{4}$ are immediately equated, and the new conservative arrangements, $A_{3}$ and $A_{4}$, replace the primitive genomes and establish a balanced polymorphism in which the two genomes are represented in each population, each at a frequency of 0.5 . Since $m_{1}=m_{2}=0.5$, the two populations are now a single population. Since the alternative genomes are adapted to separate environments, the model now simulates a population in which two different niches are recognised, and each persisting genome is separately adapted to one or other niche. The parallels between this situation and the inversion polymorphisms in Drosophila spp. are obvious.

\section{SEGOND SERIES: Without PREADAPTATION}

\section{(a) Design of experiments}

If the species is not preadapted to exploit chromosomal mutations the structural hybrids involved will be highly sterile. We may again deduce appropriate fitness coefficients on a subjective but logical basis:

(1) As in the First Series, $a=1 \cdot 0, h=1 \cdot 0, o=1 \cdot 0, t=1 \cdot 0, e=0.5$, $j=0.5, k=0.5, r=0.5, b=0.5, l=0.5$.

(2) The homogenomic heterokaryotypes, even though residing in the environment to which their genotypes maximally adapt them, will be highly infertile because of the lack of preadaptation.

Thus, say $c=0.5, q=0.5$.

The homogenomic heterokaryotypes residing in environments to which they are not well adapted will be even less fit than above.

Thus, say $g=0.3, m=0.3$.

(3) In heterogenomes, heterokaryotypy will lead to an increased sterility over and above that caused by segregational effects. Where the heterogenome is heterozygous for a single interchange, the additional sterility due to structural heterozygosity will not be as pronounced as with the doubled structural heterozygosity.

Thus, say $d=0.3, n=0.3, f=0.3, p=0.3, i=0.1, s=0.1$.

Again, eight population pairs are initiated with the migration rates and initial genome frequencies as used in the First Series, and run over one hundred consecutive generations. The results are displayed in fig. 3.

\section{(b) Results of the experiments}

Again it is verified, in populations $(3,1,1),(3,2,1),(3,3,1)$ and $(3,4,1)$ that, other things being equal, natural selection fixes the chromosome 
arrangement initially present at the highest frequency. In this case, however, it is clear that the fixation is more rapid than in the First Series. Obviously, transient chromosomal polymorphisms can be expected to persist for longer intervals if the species is preadapted to exploit the chromosomal mutations involved.

Populations $(3,1,2)$ and $(3,2,2)$ show, again, that lower intensities of migration can be balanced against selective pressures to achieve stable polymorphisms in recipient populations. At higher migration intensities, swamping of the recipient population by the donor population will result. Where a balance between migration rates is achieved (bottom row of fig. 3 ),

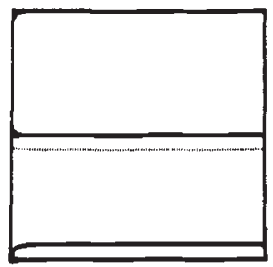

1

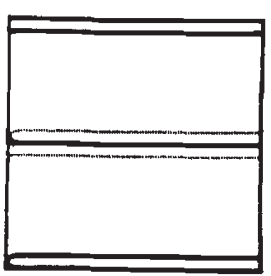

5

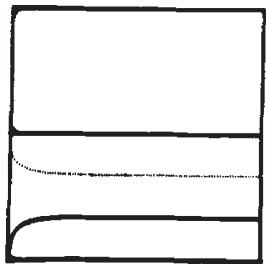

2

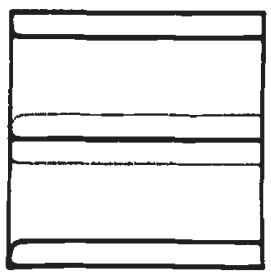

6

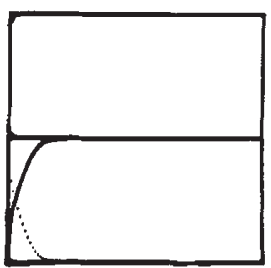

3

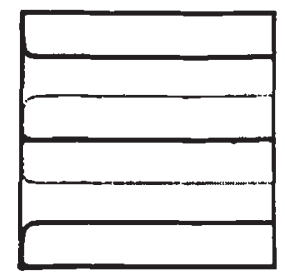

7

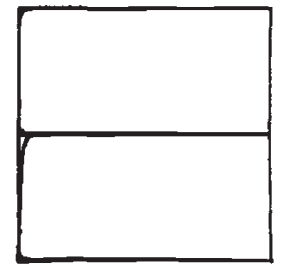

4

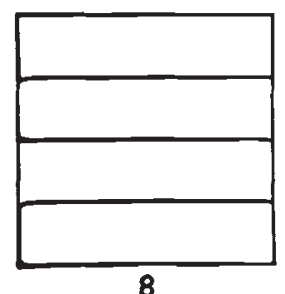

FIG. 3.-The eight episodes simulated in the Second Series. See text for fitness coefficients deduced on the basis of no preadaptation for interchange exploitation. In the top row, $m_{1}=0$; in the bottom row, $m_{1}=m_{2}$. By columns, moving left to right, $m_{2}=0.05$, $0.1,0.2,0.5$. The initial genome frequencies are $p_{1}=0.9, q_{1}=0.0, r_{1}=0.1, s_{1}=0.0$ and $p_{8}=0.0, q_{2}=0.9, r_{2}=0.0, s_{2}=0.1$.

swamping does not occur. The significant feature of this series of experiments, however, is that the replacement of the primitive genomes by the newly arisen genomes does not occur; it appears that natural selection may not elicit gross repatterning of the chromosomes directly through rearrangements for which the species is not preadapted.

\section{Third sermes: Preadapted, $A_{3}$ superadapted}

\section{(a) Design of experiments}

Say $A_{3}$ is superadapted to the environment of Population 1 , and because of this all genotypes including one or two $A_{3}$ genomes existing in Population 1 have been given an increment of 0.1 to their fitness coefficient. The array of fitness coefficients deduced, then, are as for the First Series, except that $c=1 \cdot 0, f=0.8, h=1 \cdot 1$ and $i=0.9$. The migration rates and initial genome frequencies are as for the First and Second Series. The results are displayed in fig. 4.

\section{(b) Results of the experiments}

Populations $(4,1,1),(4,2,1),(4,3,1)$ and $(4,4,1)$ are identical and verify that natural selection will elicit the fixation of the genome most fit 
in that environment. The replacement of $A_{1}$ by $A_{3}$ in these populations leads to correlated responses in migrationally interacting populations. In population $(4,1,2)$ where the immigration rate is relatively low at $m=0.05$, a balance between the immigration rate and selection pressures is established. The conservative effect of $A_{3}$ in Population 2 (i.e. in population $(4,1,2)$ ), enhances its adaptation, relative to $A_{1}$, such that it becomes, by the hundredth generation, the most frequent foreign genome in the recipient population although its migrational influx is actually less than that of $A_{1}$. Also, the conservative effect of $\mathrm{A}_{3}$ in this population enhances the fitness of the primitive native genome $A_{2}$ relative to $A_{4}$ and consequently delays the replacement, induced by the critical migration intensities, of $A_{2}$ by $A_{4}$ (compare the graphs $(4,2)$ and $(1,2))$.

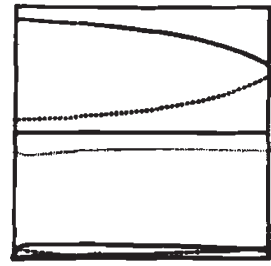

1

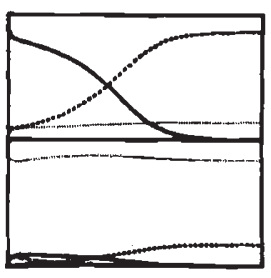

5

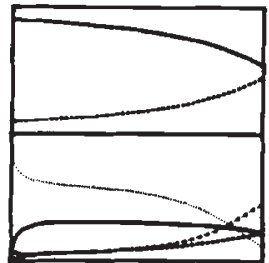

2

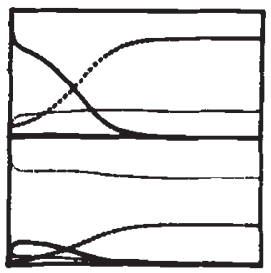

6

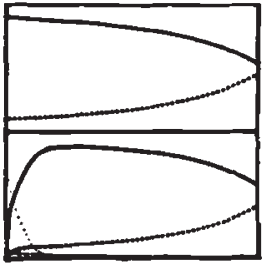

3

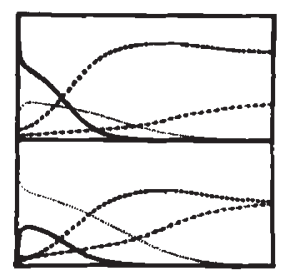

7

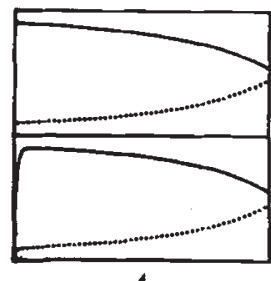

4

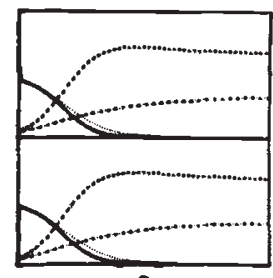

8

Fig. 4.-The eight episodes simulated in the Third Series. See text for fitness coefficients deduced on the basis of preadaptation for interchange exploitation and with increased adaptation for a novel genome in Population 1. In the top row, $m_{1}=0$; in the bottom row, $m_{1}=m_{2}$. By columns, moving left to right, $m_{2}=0.05,0.1,0.2,0.5$. The initial genome frequencies are $p_{1}=0.9, q_{1}=0.0, r_{1}=0.1, s_{1}=0.0$ and $p_{2}=0.0, q_{2}=0.9$, $r_{1}=0.0, s_{2}=0.1$.

At higher migration intensities, swamping occurs such that the recipient population is absorbed by the donor. The replacement of $A_{1}$ by $A_{3}$ in the captured population follows as a correlated response to the replacement of $A_{1}$ by $A_{3}$ in Population $\mathrm{l}$, even though $A_{3}$ is not more adapted to the environment of Population 2 than is $A_{1}$.

Where migration operates in both directions (graphs $(4,5),(4,6),(4,7)$ and $(4,8)$ ) the influx of $A_{2}$ genomes into Population 1 enhances the fitness of the already superadapted $A_{3}$ genome and causes a more rapid replacement of $A_{1}$ by $A_{3}$. Again, correlated responses are induced in Population 2. In all cases, a stable situation is ultimately achieved. The time taken to achieve this stability, the nature of the transient polymorphic populations, and the constitution of the stable populations are, of course, functions of the migration rates and selection coefficients operative. Furthermore, all these possible outcomes are subject to the effects of initial genome frequencies as indicated in the fourth series of experiments. 


\section{Fourth series: higher initial genome FREQUencies}

(a) Design of experiments

The population simulations of the first three series in which $m_{2}=0.1$ or 0.2 were repeated, but in each case, values $q_{2}=0.8$ and $s_{2}=0.2$ were used.

\section{(b) Results of the experiments}

The higher initial frequency of $A_{4}$ in Population 2 lead to some perhaps unexpected results as well as to quite expected ones. Some of these perhaps unexpected results are illustrated in fig. 5 . Thus, graph $(5,1)$ which should be contrasted with $(2,7)$ shows that the higher initial frequency of $A_{4}$ in Population 2 induces a more rapid replacement of $A_{2}$ by $A_{4}$ in that population, leading to the establishment of $A_{4}$ at particularly high levels. This, in

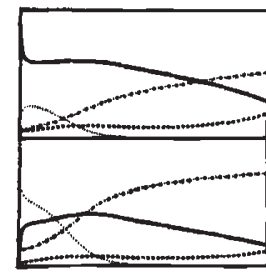

1

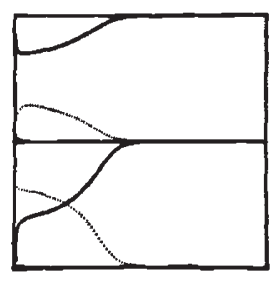

2

Fig. 5.-Two of the evolutionary episodes simulated in the Fourth Series. See text for fitness coefficients deduced: in the left-hand pair, on the basis of preadaptation; in the righthand pair, on the basis of no preadaptation for the exploitation of interchange. In each case, $m_{1}=m_{2}=0.2$, and the initial genome frequencies are $p_{1}=0.9, q_{1}=0.0$, $r_{1}=0.1, s_{1}=0.0$ and $p_{2}=0.0, q_{2}=0.8, r_{2}=0.0, s_{2}=0.2$ (i.e. higher initial frequency of the novel genome in Population 2).

turn, induces the correlated response in the migrationally interacting Population 1 such that $A_{4}$, for a period at least, becomes the most abundant genome in that population. Secondly, graph $(5,2)$ which should be compared with $(3,7)$ shows that the higher initial frequency of $A_{4}$ in Population 2 destroyed the balance which obtained in $(3,7)$ and caused the swamping of one population by the other. And, it is the most frequent genome in the population with the smallest genetic load $\left(A_{1}\right)$ which takes over.

\section{Discussion}

The model described is indeed capable of simulating a variety of situations of biological interest, and among other things, it suggests that under appropriate conditions, new chromosomal arrangements may be incorporated into a gene pool by the action of natural selection.

Although it is probable that the whole array of possible mutational events is available to all higher organisms, the probability that any particular mutation will be exploited will depend upon many factors, one of which embraces the concept of preadaptation. Preadaptation is achieved through the incorporation of those adjustments into the biology of the organism which minimise the sterilising effect of the structural hybridity, and a species must be preadapted if it is to exploit the chromosome mutation concerned. 
Evolution is the result of cumulative changes wrought by the unrelenting selection of the best adapted systems, generation upon generation, and the adjustments incorporated into the biology of the organism which constitute preadaptation for a particular cytoevolutionary tactic must themselves be incorporated through natural selection. The terminal localisation of chiasmata, which makes interchange exploitation possible, must have evolved, in those taxa which have exploited interchanges, as a response to some more ancient selective pressure. For example, the cytological preadaptation of Isotoma petraea for interchange exploitation which has culminated in the evolution of complex hybridity (James, 1965, 1970) probably arose as an adaptive adjustment in a genetic system in which an innovative breeding mechanism, viz. the ancestral Lobeliaceous crosspollination mechanism, was balanced against a conservative meiotic system, in much the same way as comparable balances have been achieved in Allium (Ved Brat, 1965) and elsewhere.

If the population is not preadapted for the exploitation of a particular structural change, natural selection alone can perhaps never directly elicit the fixation of chromosomal barriers of this type between two populations. However, if we consider three populations, 1,2 and 3 , the $1 / 2$ and $2 / 3$ interactions may separately lead to the fixation of structural differences for which the populations are preadapted, and so construct a state whereby 1 and 3 are differentiated for structural differences beyond the scope of their preadaptation. For example, natural selection may fix two interchange differences between populations 1 and 2 and between populations 2 and 3 . In the $1 / 2$ and $2 / 3$ interpopulational heterozygotes, $2 \odot_{4}$ and/or $\odot_{6}$ progeny may result. Preadaptation of the population may well permit the occurrence of such small rings of high fertility. On the other hand, the $1 / 3$ interpopulation heterozygote may be a $\bigodot_{10}$ and the non-disjunction associated with this ring may negate the advantages associated with the maintenance of adapted gene arrays ( $c f$. James, 1969). In other words, although the populations 1, 2 and 3 are all preadapted to exploit interchanges, this preadaptation extends only to small rings. However, by exploiting these small rings, it is clearly possible for natural selection to construct multiple interchange barriers between potentially interacting populations.

Since chromosome structural change arises through mutation, it occurs initially in the heterozygous condition, imparting a measure of sterility upon its carrier even if preadaptation obtains. If the structural change is to persist in the population, it must be associated with some selectively advantageous quality of magnitude sufficient to compensate for the decreased fertility of the heterozygote. Although it is possible that a new chromosomal arrangement may be associated with a novel and adaptively superior molecular sequence, it is more than likely that the value of such a rearrangement depends upon its control over recombination. For such heterokaryotypes to compete effectively against homokaryotypes, they must perform better as parents; their fitness resides in their reduced genetic load (Darlington, 1956; Wallace, 1959; Lewis and John, 1967). This situation is possible only in organisms hybrid for supergenic arrays whose integrity tends to be preserved by the chromosomal hybridity. It is this situation which is simulated by the model described in this paper and it is clear that under critical intensities of migrational interaction chromosome structural changes, for which the species is preadapted, may be fixed by natural 
selection. These critical intensities of migrational interaction provide the conditions under which the chromosomal changes become adaptive.

Furthermore, it is clear that in situations where novel genomes are abruptly raised to high frequencies, significant and perhaps unpredictable responses, and correlated responses, may occur amongst migrationally interacting populations. Indeed, this situation may simulate, in a naive way, the "genetic revolution" emanating from the shift towards inbreeding associated with the "Founder Principle" (Mayr, 1942, 1954), with " catastrophic selection" (Lewis, 1962) and with " intrademe selection" following the ascent of new peaks in the "adaptive surfaces" envisaged by Wright (1964).

Finally, it is of interest to note the magnitude of these critical intensities of migrational interaction. Current influential opinion, e.g. Simpson (1953) and Mayr (1963), generally agrees that migration rates greater than about 0.01 will unite interacting populations into an effectively single entity. This model suggests otherwise; the critical intensities for chromosome repatterning are apparently those one might well expect to be operating between Wrightian demes. Since the fixation of any conservative difference must contribute to an ultimate cleavage of the gene pool, the implications of this model to the concept of sympatric speciation warrant careful examination.

\section{SUMMARY}

1. A model which simulates a variety of situations of cytoevolutionary interest is described.

2. Very high migrational intensities, if unbalanced, lead to a swamping of recipient populations; low migrational intensities lead to a balance between selection pressures and migration pressures; intermediate migrational intensities of the order of 0.1 and greater elicit the fixation of those devices (e.g. interchanges) which tend to conserve the significant differences between populations.

3. Preadaptation of a population is necessary before natural selection can exploit any particular conservative device.

Acknowledgments.-The author thanks the Director and Staff of the Computing Center, University of Western Australia, for their generous help and co-operation, and the Australian Research Grants Committee for supporting the associated research.

\section{REFERENGES}

DARLINGTon, C. D. 1958. The Evolution of Genetic Systems (2nd ed.). Oliver \& Boyd, Edinburgh.

JAMEs s. H. 1965. Complex hybridity in Isotoma petraea. I. The occurrence of interchange heterozygosity, autogamy and a balanced lethal system. Heredity, 20, 341-353.

JAMEs, s. H. 1970. Complex hybridity in Isotoma petraea. II. Components and operation of a possible evolutionary mechanism. Heredity, 25, 53-77.

LEWIS, H. 1962. Catastrophic selection as a factor in speciation. Evolution, 16, 257-271.

LEWIS, K. R., AND B. JOHN. 1967. Chromosome variability and geographic distribution in insects. Science, 152, 711-721.

MAtHER, K. 1943. Polygenic inheritance and natural selection. Biol. Rev., 18, 32-64.

MAYR, z. 1942. Systematics and the Origin of Species. Columbia University Press, New York. MAXR, E. 1954. Change of genetic environment and evolution. In J. Huxley, A. C. Hardy, and E. B. Ford, Eds., Evolution as a Process (Allen and Unwin, London), 157-180.

MAYR, z. 1963. Animal Species and Evolution. Oxford University Press, London. 
simpson, G. G. 1953. The Major Features of Evolution. Columbia University Press, New York.

VED BRAT, s. 1965. Genetic systems in Allium. III. Meiosis and breeding systems. Heredity, 20,325-339.

WALlaCE, B. 1959. The influence of genetic systems on geographic distribution. Cold Spring Harbor Symp. Quant. Biol., 24, 193-204.

wright, s. 1964. Stochastic processes in evolution. In J. Gurland, Ed., Stochastic Processes in Medicine and Biology (University of Wisconsin Press, Madison), 199-241. 